\title{
Range Resolution Improvement in Passive Coherent Location Radar Systems using Multiple FM Radio Channels
}

\section{A Sinan Tasdelen \& H Köymen, Bilkent University, Turkey}




\begin{abstract}
With the improvement in digital signal processor technology, the topic of Passive Coherent Location (PCL) radar systems that exploit 'illuminators of opportunity' became a popular area of research. Comparative studies of Ambiguity function properties of broadcast signals such as FM radio, analogue television, cellular phone signals and digital audio broadcast (DAB) revealed that FM radio broadcast signals, providing up to $30 \mathrm{~dB}$ peak to side lobe levels in range, and up to $40 \mathrm{~dB}$ peak to side lobe levels in Doppler, are well suited for passive radar purposes.
\end{abstract}

However, due to low modulation bandwidth in FM, which never reaches the allowable channel bandwidth of $150 \mathrm{kHz}$, the range resolution is severely limited. While $150 \mathrm{kHz}$ modulation bandwidth can provide $1 \mathrm{~km}$ range resolution, at best, $30 \mathrm{kHz}$ modulation bandwidth is achieved, which results in 5km range resolution. Furthermore, the modulation bandwidth is strongly dependent on the content that is being broadcasted.

An improvement in range resolution is obtained by using multiple adjacent FM channels, emitted from co sited transmitters, which is often the case in large towns in countries, where the FM channel allocations are weakly regulated. A number of channels can be used independently or together. When channels are used together the range resolution is dominated by the total channel bandwidth. The auto ambiguity function of multiple channel FM signals shows high side lobes. Once the range is estimated using a single FM signal, it can be refined by using more FM channels subsequently. The number and locations of the FM channels that will be used in the PCL process can either be predetermined or chosen at each recording separately, taking into account the power and modulation bandwidth of each channel.

A new scheme is proposed, which takes multiple FM radio channels emitted from co sited radio transmitters.

Again two receiver antennas are used: One for direct signal reception and one for the target scattered signal reception. The direct signal is used in the computation of the autocorrelation function of the FM waveform. The signals from both antennas are used in the computation of the cross ambiguity function. The cross ambiguity function is compared to the autocorrelation function. The range information is obtained by the delay between the cross ambiguity function and the auto ambiguity function. Simulations show 
that down to $-30 \mathrm{~dB}$ signal to noise ratio (SNR) the autocorrelation function of 7 adjacent FM channels with random content can be successfully extracted from the cross ambiguity function. Hence, the range resolution is significantly improved. Best results are achieved when all channels have equal power. For sufficiently large SNR, the cross ambiguity function for multi FM channel waveform has an envelope similar to the cross ambiguity function a singular FM channel waveform. Range resolution can be improved down to 180 meters when 7 adjacent FM channels are used together. 


\title{
Range Resolution Improvement in Passive Coherent Location Radar Systems Using Multiple FM Radio Channels
}

\author{
A.S. Taşdelen, H. Köymen \\ Department of Electrical and Electronics Engineering at Bilkent University, Ankara, Turkey \\ sinan@ee.bilkent.edu.tr, koymen@ee.bilkent.edu.tr
}

Keywords: Passive coherent location (PCL), range resolution, modulation bandwidth, FM radio, cross-ambiguity

\begin{abstract}
Passive coherent location (PCL) radar systems that use single FM radio channel signal as illuminator of opportunity have limited range resolution due to low modulation bandwidth and high dependence on the content that is being broadcasted from the FM station. An improvement in range resolution is obtained by using multiple adjacent FM channels, emitted from co-sited transmitters, which is often the case in large towns in countries, where the FM channel allocations are relatively weakly regulated. The proposed scheme computes the autocorrelation function of the signal directly received from the FM co-located transmitter, and compares it to the cross-ambiguity function, obtained from direct and target scattered signals. The geometry of the problem is like in the case of monostatic radar. The range information is obtained by the delay between the cross-ambiguity function and the autocorrelation function. It is shown that down to $-37 \mathrm{~dB}$ signal to noise ratio (SNR) the autocorrelation function of 7 FM channels with different contents can be successfully extracted from the cross-ambiguity function. The detection of the time delays is a linear estimation problem. The issue of time-delay estimation is a known topic of research. A powerful estimator can be found.
\end{abstract}

\section{Introduction}

The recent improvement in digital signal processor technology made the topic of Passive Coherent Location (PCL) radar systems that exploit 'illuminators of opportunity' a popular area of research, again.

Comparative studies of Ambiguity function properties of broadcast signals such as FM radio, analogue television, cellular phone signals and digital audio broadcast (DAB) revealed that $\mathrm{FM}$ radio broadcast signals, providing up to $30 \mathrm{~dB}$ peak to side lobe ratio in range, and up to $40 \mathrm{~dB}$ peak to side lobe ratio in Doppler, are well suited for passive radar purposes $[1,2]$.

However, in [2] it is shown that the modulation bandwidth of a single FM radio channel is only a fraction of the whole bandwidth, $150 \mathrm{kHz}$, allocated to that channel. Furthermore, modulation bandwidth depends strongly on the content that is being broadcasted from that station. It is reported that the bandwidth is seen to vary between $500 \mathrm{~Hz}$ and $22.2 \mathrm{kHz}$, where highest bandwidth is obtained when fast tempo jazz is played as content. The range resolutions obtained from these signals is in the range of 6757 meters to $300 \mathrm{~km}$.

FM radio based PCL radar systems are shown to detect and track targets to ranges up to $150 \mathrm{~km}$ from the receiver [3].

\section{Using Multiple FM Radio Channels}

The range resolution in radar systems is inversely proportional to the bandwidth of the waveform that is being used. This relation suggests that range resolution in PCL systems that use FM radio broadcast as illuminator of opportunity' can be improved by increasing the total modulation bandwidth of the waveform. This can be done by making use of the signals of as many channels as required to achieve the aimed range resolution.

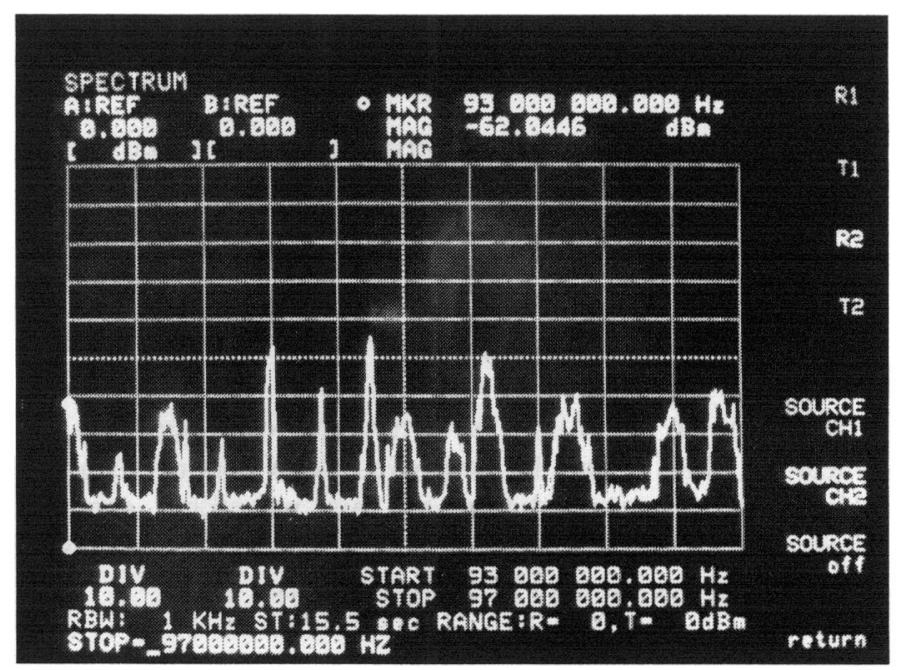

Figure 1: Part of FM frequency spectrum recorded at Bilkent University, Ankara. Signals are transmitted from Çaldağı, Ankara

Unlike in analogue TV broadcast signals, where the separation of each carrier frequency is very difficult [4], FM carriers are inherently separated. The 87.5-108 $\mathrm{MHz}$ frequency spectrum is allocated to FM radio broadcast. In one 
service area, the carriers are separated at least by $300 \mathrm{kHz}$. In countries, where the FM radio channel allocations are weakly regulated, the maximum frequency deviation of the channels are faintly controlled, and frequencies that fall into the adjacent channel are not adequately suppressed, causing in metropolitan towns spectrum readings as seen in Figure1.

Interferences between adjacent channels, as seen in Figure 1, although not desired in telecommunication, are beneficial for the proposed system. For instance, in Figure 1, the whole 94.7-95.6 MHz spectrum or 96.5-97 $\mathrm{MHz}$ spectrum can be made use of, which has larger bandwidth than a single FM channel, yielding a smaller range resolution.

\subsection{Ambiguity function of single and multi-channel FM Waveforms}

The radar ambiguity function is a long used measure in evaluating the performance of a waveform in radar applications. It represents the output of a matched filter [5], and is given by the expression

$$
|A(\tau, v)|^{2}=\left|\int_{-\infty}^{\infty} s(t) s^{*}(t+\tau) \exp (j 2 \pi v t) d t\right|^{2}
$$

where $A(\tau, v)$ is the ambiguity response at delay $\tau$ and Doppler $v$ and $s(t)$ is the signal under consideration. It can be shown that the ambiguity function has a peak at $(0,0)$ and the extent of this peak in delay (or range) and Doppler determines the range and Doppler resolutions respectively [6]. It can be shown that this peak is a global maximum for the ambiguity function. When $v=0$ ambiguity function reduces to the autocorrelation function.

The ambiguity function of a frequency modulated signal is given in Figure 2. Since the ambiguity function is symmetric for this signal, only a quarter is plotted. The message signal is a $15 \mathrm{kHz}$ filtered monophonic music file of $100 \mathrm{msec}$ in length. The FM signal has a maximum frequency deviation of $\pm 75 \mathrm{kHz}$ with no pre-emphasis.

The Doppler resolution is a function of integration time, meaning that it can be improved with longer recordings. However, range resolution of this waveform cannot be improved without changing its bandwidth or waveshape, which is not possible in PCL applications.

The ambiguity function of the signal containing three adjacent FM channels, having different monophonic contents and having $100 \mathrm{kHz}$ equally spaced carrier frequencies, is depicted in Figure 3. It is observed that the ambiguity function of the signal containing a single FM channel is an envelope to the ambiguity function of three FM station signal.

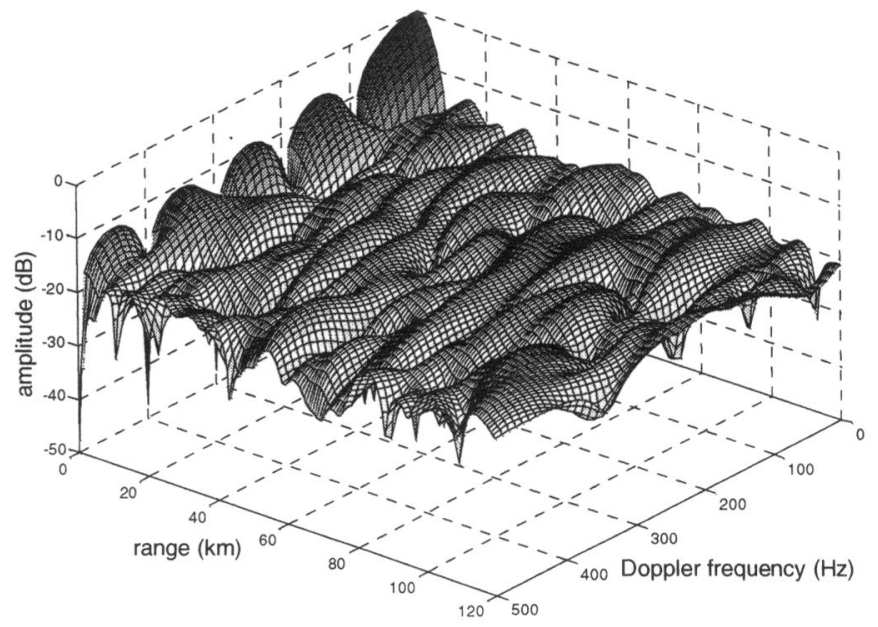

Figure 2. Ambiguity function of single FM channel signal

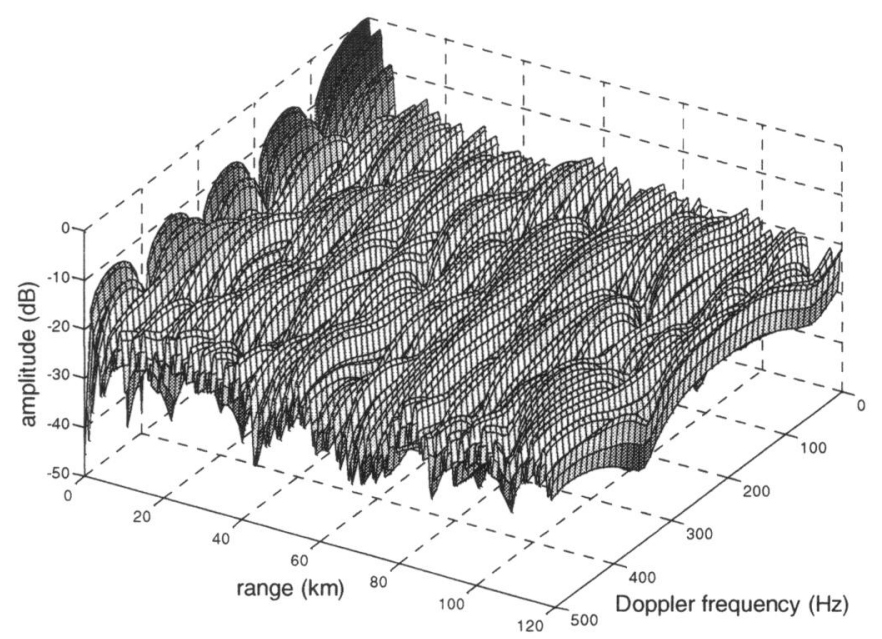

Figure 3. Ambiguity function of 3 adjacent FM channels signal

The range resolution obtained by using only one FM channel, and the range resolution obtained from three adjacent stations that broadcast different content are depicted in Figure 4. Both signals have message contents with similar bandwidth.

The envelope of the autocorrelation function for three adjacent FM stations is the autocorrelation function for a single FM station. This is due to the fact that the spectrum of three adjacent FM station signals is almost like a $100 \mathrm{kHz}$ repetition of a single FM station spectrum. Due to the periodic structure in the spectrum, a sinc type formation is observed in the range resolution plot. Since the modulation bandwidth is about three times the bandwidth of a single FM transmission, the peak in the autocorrelation function is narrower. 


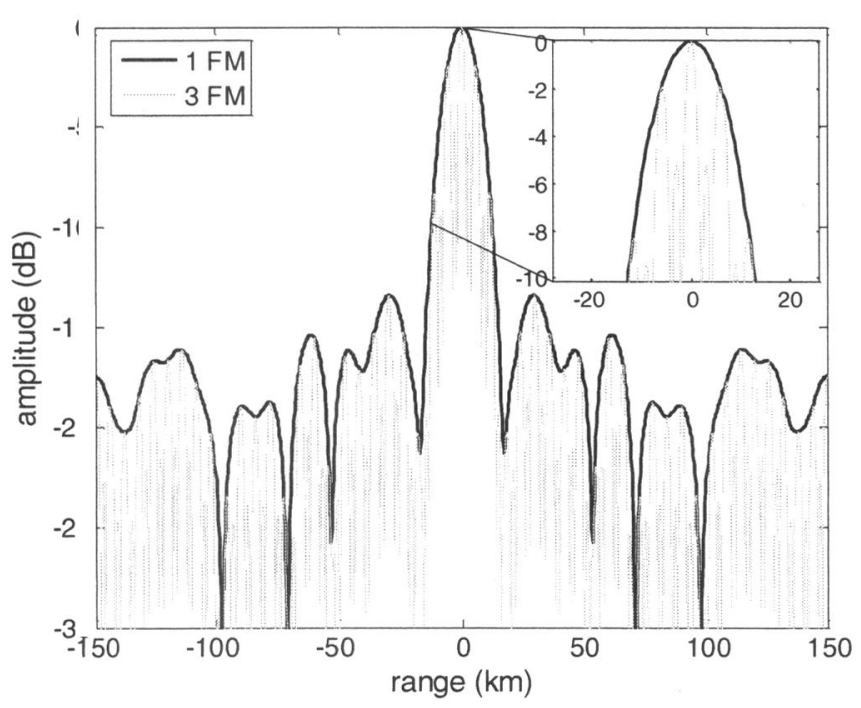

Figure 4. Autocorrelation functions of single FM channel waveform and three adjacent FM channels waveform

The peak can be made narrower when seven adjacent stations are used together (Figure 5).

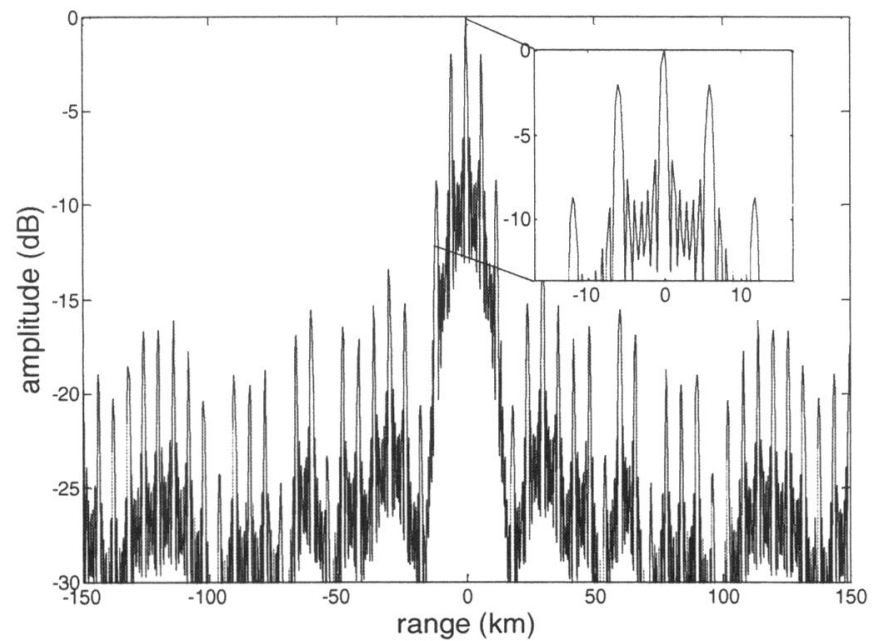

Figure 5. Autocorrelation function for seven adjacent FM stations

As in the three FM station case, the shape of the autocorrelation function of the waveform from seven adjacent FM stations resembles the autocorrelation plot of a single FM transmission. However some side-lobes are increased. Again, a sinc type formation is observed in the range resolution plot. If stations are selected in a random way so that they are not adjacent, then the autocorrelation starts to lose its resemblance to the autocorrelation function of a single FM waveform. The autocorrelation function of seven FM signals having equal power, but distributed randomly in $1500 \mathrm{kHz}$ bandwidth with minimum separation of $150 \mathrm{kHz}$ and maximum separation $250 \mathrm{kHz}$ is depicted in Figure 6.

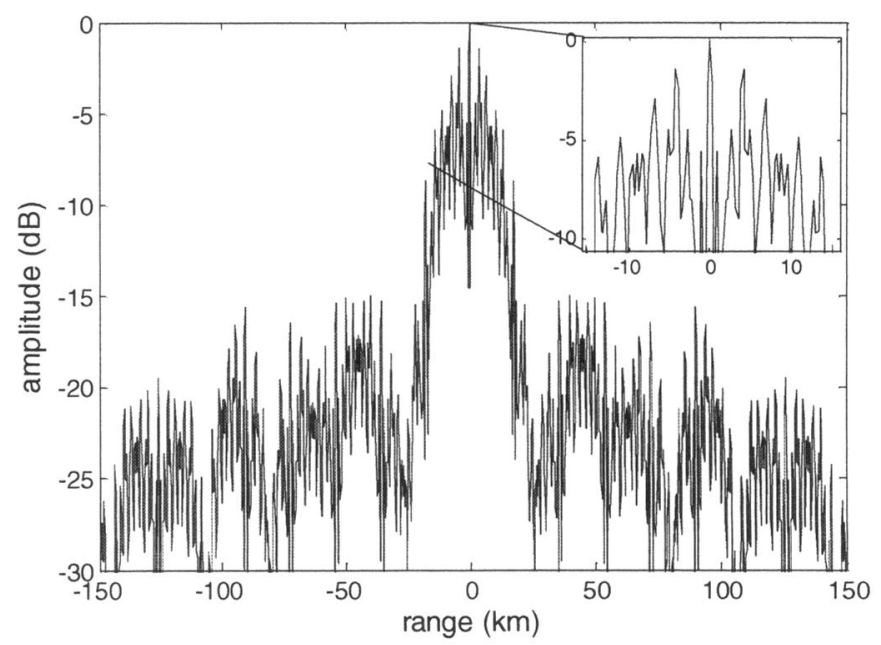

Figure 6. Autocorrelation function for seven non-adjacent FM stations

As expected, the sinc type formation in the range resolution is lost due to the failed periodicity and the side-lobes in the vicinity of the peak are increased. However, the main peak is still narrow. Theoretically, the range resolution can be improved down to $2 \mathrm{~km}$ with an appropriate detection algorithm.

\subsection{Minimum SNR Criterion}

In the analysis of the auto-ambiguity and autocorrelation functions the scattered signal is assumed to be noise free. As the received power of the scattered signal becomes smaller, the SNR decreases for far away targets. Consequently, the ambiguity function deteriorates, yielding a worse range resolution or even target loss or false alarm.

The proposed detection method depends on the minimum SNR that allows a successful detection of the autocorrelation function, buried in the cross-correlation. This is discussed in Section 3 of this paper.

In the following simulations, the receiver noise is taken as white and Gaussian distributed, with spectral height of $\mathrm{kT}_{0} \mathrm{~B}$, where $\mathrm{k}$ is the Boltzmann constant, $\mathrm{T}_{0}$ is the temperature in Kelvin (taken as $300^{\circ} \mathrm{K}$ ) and $\mathrm{B}$ is the bandwidth. The bandwidth is $200 \mathrm{kHz}$ in single FM signal simulation and 800 $\mathrm{kHz}$ in seven FM signals simulation, where the carriers are spaced at multiples of $100 \mathrm{kHz}$, and signal powers are equal. The signals are sampled at $5 \mathrm{MHz}$. Simulations show that for a single FM signal, the autocorrelation function preserves its shape down to $-19 \mathrm{~dB}$ SNR. For lower SNR the crosscorrelation terms between signal and noise start to dominate. However, the peak of the autocorrelation is dominant down to $-29 \mathrm{~dB}$ as shown in Figure 6. If the number of FM signals is increased, the autocorrelation shape shows no variation down to $-6 \mathrm{~dB}$. The changes until $-26 \mathrm{~dB}$ are small and on the sidelobes. At $-36 \mathrm{~dB}$ the peak is no more dominant over the sidelobes (Figure 7). 


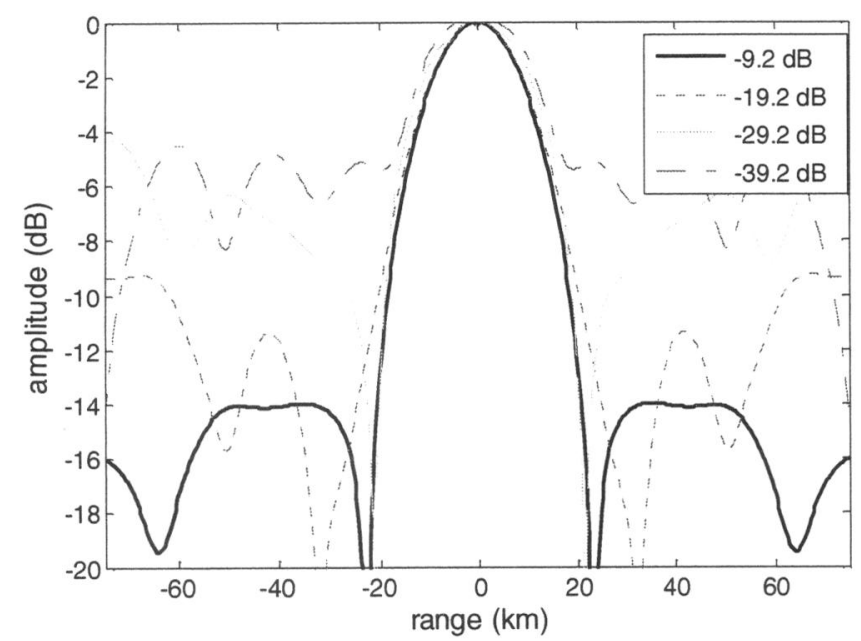

Figure 7. Autocorrelation function for single FM signal for various SNR

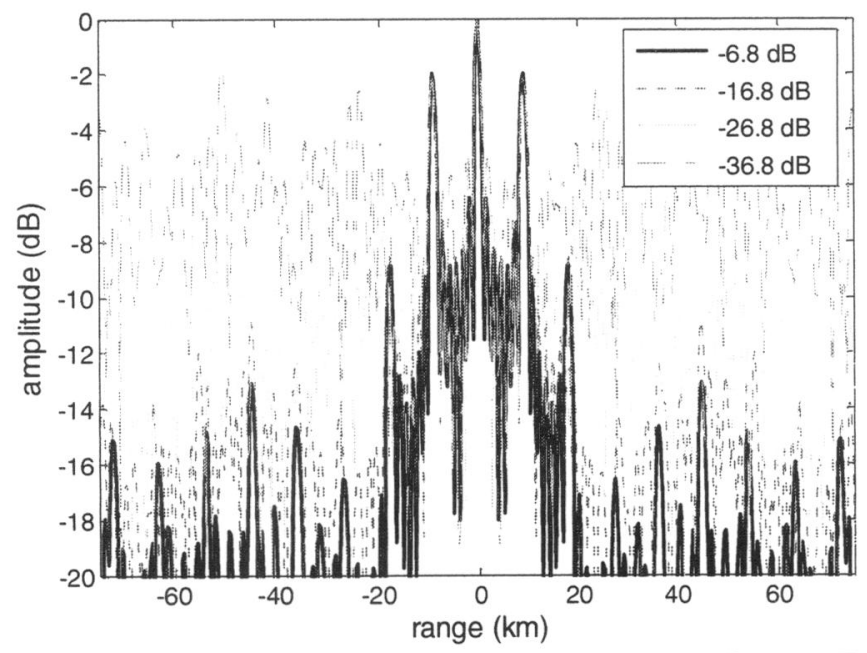

Figure 8. Autocorrelation function for seven adjacent FM signals for various SNR

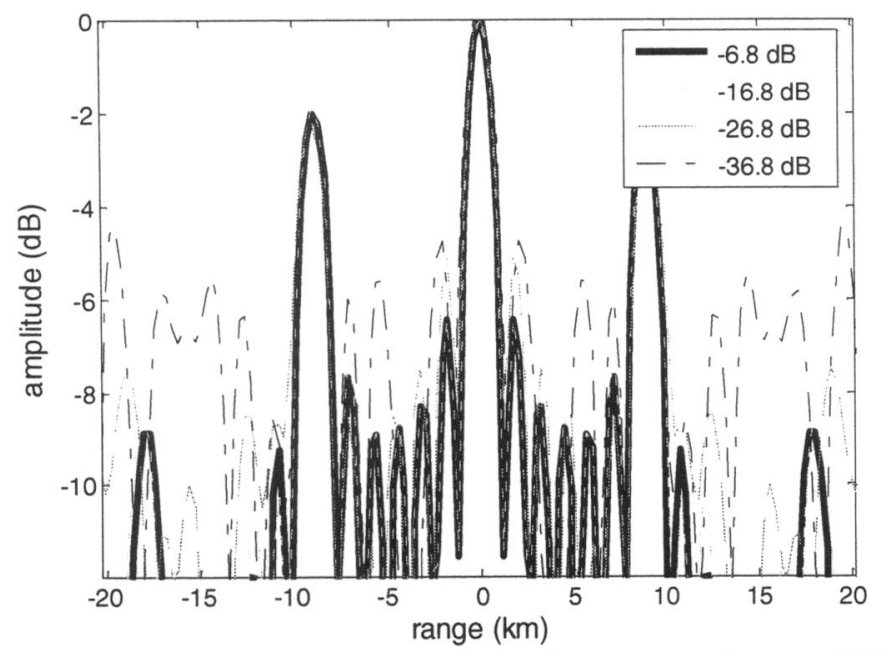

Figure 9. Autocorrelation function for seven adjacent FM signals for various SNR (zoomed)

\section{Detection}

In [7] it is shown that the Doppler resolution of a PCL system, using FM radio broadcast as non-cooperative illuminator, is typically $1 \mathrm{~Hz}$, corresponding to a velocity resolution of around $1.5 \mathrm{~ms}^{-1}$. The Doppler resolution can be improved by taking a longer integration time. In the analysis below it is assumed that the Doppler shift of the target is found by the radar and the signal is perfectly corrected in frequency.

Assume $\mathrm{s}(\mathrm{t})$ is broadcasted from a FM radio transmitter and received from a co-located receiver. This direct signal, $s(t)$ at the receiver has a high SNR. The signal reflected from $\mathrm{N}$ targets and received from the receiver is represented by

$$
s_{N}(t)=\sum_{i=1}^{N} \alpha_{i} s\left(t-t_{i}\right)+n(t)
$$

where $\alpha_{\mathrm{i}}$ is the attenuation constant for the signal scattered from the $\mathrm{i}^{\text {th }}$ target, $\mathrm{t}_{\mathrm{i}}$ is the two-way time delay of the signal scattered from the $\mathrm{i}^{\text {th }}$ target and $\mathrm{n}(\mathrm{t})$ is the zero mean white Gaussian noise process. The result of the cross-correlation of direct and target scattered signals is given by

$$
C(\tau)=\sum_{i=1}^{N} \alpha_{i} R\left(\tau-t_{i}\right)+n s(\tau)
$$

where $\mathrm{A}$ is the autocorrelation of the direct signal and $\mathrm{ns}$ is the cross-correlation of the direct signal and noise. Taking the Fourier transform of the cross-correlation function, we obtain

$$
C(f)=\sum_{i=1}^{N} \alpha_{i} \exp \left(-j 2 \pi f t_{i}\right) F\{R(\tau)\}+N_{0} S(f)
$$

where $\mathrm{F}\{\mathrm{R}(\tau)\}$ is the Fourier transform of the autocorrelation function, $N_{0}$ is the power spectral density of the white noise and $S(f)$ is the Fourier transform of the direct signal. Dividing both sides by $\mathrm{A}(\mathrm{f})$ and applying the autocorrelation property of the Fourier transform we obtain

$$
\begin{gathered}
\frac{C(f)}{|S(f)|^{2}}=\sum_{i=1}^{N} \alpha_{i} \exp \left(-j 2 \pi f t_{i}\right)+\frac{N_{0} S(f)}{|S(f)|^{2}} \\
=\sum_{i=1}^{N} \alpha_{i} \exp \left(-j 2 \pi f t_{i}\right)+\frac{N_{0}}{S^{*}(f)}
\end{gathered}
$$

where $*$ denotes the complex conjugation. The problem reduces to finding the time shifts $t_{i}$ in the exponential. This is a linear estimation problem, and very good estimators are available. In the estimation problem for single channel FM signal, the estimation is done around one frequency, whose width determines the range resolution. The SNR around this frequency is higher than in other frequencies. If, for instance, 3 FM channels are used, the estimation can be done around 
three frequencies, which implies that the range resolution is improved. The total signal power increases, but due to the additional bandwidth the noise power increases too. The total SNR, therefore, does not change. This results in the same detection performance as in the single FM channel case.

\section{Conclusions}

In this paper, we have shown that by using multiple FM broadcast channels as the 'illuminator of opportunity' for a PCL radar system, the range resolution, which is in the order of 6-7 km for highest modulation bandwidth measurements for single channel FM transmission, can theoretically be improved down to $1 \mathrm{~km}$ with seven channel FM signals. The estimation around multiple frequencies suggests that the range resolution can actually be further improved. The minimum SNR criterion in the finding of the autocorrelation function in the cross-correlation is important. For low SNR this issue becomes problematic.

We will handle the issue of an optimum detection and location algorithm, which finds the time shifts $t_{i}$ in equation (6), as a future work. We will test efficient algorithms for time delay estimation like in [8] for their performance.

We will also evaluate the performance of first-detect-thenlocate algorithms that use single FM channel signal for fast target detection, and multiple FM channel signals for finer localization of a target and/or distinction of multiple close targets.
We will investigate the $\mathrm{Bi}$-static nature of the problem as a future work.

\section{References}

[1] H.D. Griffiths, C.J. Baker. "Passive coherent location radar systems. Part 1: Performance prediction", IEE Proc., Radar Sonar Navig., 152, pp. 153-159, (2005).

[2] C.J. Baker, H.D. Griffiths, I. Papoutsis. "Passive coherent location radar systems. Part 2: Waveform properties", IEE Proc., Radar Sonar Navig., 152, pp. 160-168, (2005).

[3] P.E. Howland, D. Maksimiuk, G. Reitsma. "FM radio based bistatic radar", IEE Proc., Radar Sonar Navig., 152, pp. 107-115, (2005).

[4] P.E. Howland. "Target tracking using televisionbased bistatic radar", IEE Proc., Radar Sonar Navig., 146, pp. 166-174, (1999).

[5] B.R. Mahafza. 'Radar systems analysis and design using MATLAB', (Chapman \& Hall/CRC, 2000), Chap 6.

[6] N. Levanon, E. Mozeson. 'Radar Signals', (John Wiley \& Sons, 2004), Chap.3.

[7] P.E. Howland, D. Maksimiuk, G. Reitsma. "FM radio based bistatic radar", IEE Proc., Radar Sonar Navig., 152, pp. 107-115, (2005).

[8] J. Li, R. Wu, "An efficient algorithm for time delay estimation”, IEEE Trans. Signal Proccessing, 46, pp. 2231-2235, (1998) 